\section{9c OCCUPATIONAL HEALTH SERVICES IN ROMANIA AND IN EASTERN EUROPE}

Carmen Iliana Busneag. Occupational Medical Department, National Romanian Television, Bucharest, Romania

\subsection{6/oemed-2018-ICOHabstracts.639}

The Southern Eastern (SE) Europe with its 14 countries currently has approximately 68 million inhabitants, of which more than 18 million are workers. In the last two decades, the countries from SE Europe faced increasing needs to develop and adapt the occupational health policies, systems and services to new conditions. Establishment of democracy and market economy leaded to the new principles of managing Occpational Health Services (OHS), development of new health and safety legislation aligning to the requirement of the EU acquis communautaire and establishment of modern $\mathrm{OSH}$ in the region.

In Romania, the National Occupational Safety and Health Strategy 2016-2020 is the framework instrument to allow articulation with the EU strategic guidelines on health and safety at work. The basic principle is fair access to health care services, cost-effectiveness, substantiation on evidence, optimisation of health services, focusing on the detection of diseases generated or aggravated by working conditions, as well as preventive services and interventions, partnering with all actors that can contribute to improving health at work.

During 2007-2015 period the following guides were launched:

- Special medical surveillance for workers professionally exposed to ionising radiation,

- General aspects of industrial toxicology; methods of analysis used in industrial toxicology,

- Guidance on occupational exposure to asbestos,

- Terms and concepts of industrial toxicology and work psychology.

SWOT analysis indicates STRONG POINTS as 1) legislation on safety and health at work has been imposed in current practice in all sectors of activity, both public and private, 2) the assistance of occupational medicine is granted only to specialists in occupational medicine, and WEAKNESS as 1) the limitation of medical services predominantly to health assessment actions, to the detriment of health prevention, 2) underreporting of occupational diseaseas and occupational related diseases. This is a major problem of all countries in SE Europe!

\section{$1719 \mathrm{~d}$ THE CURRENT STATUS OF OCCUPATIONAL HEALTH SERVICES AND ITS PERSPECTIVES IN KOREA}

Eun A Kim, Jang-jin Ryoo. Korea Occupational Safety and Health Agency (KOSHA), Ulsan, Republic of Korea

\subsection{6/oemed-2018-ICOHabstracts.640}

The occupational health services have been developed and expanded continuously in Korea as government policy and the social demand on occupational health been changed. The $\mathrm{OH}$ system of Korea is based on a triangle system which consists of government (MoEL), Korea Occupational Safety and Health Agency (KOSHA) and the private institutions. The occupational health services are conducted by experts in various fields such as occupational physicians, nurses, hygienists, ergonomists etc. The occupational health services in Korea were concentrated in the manufacturing field of machinery, automobile and shipbuilding so far, and it is expanding into the services and the construction fields. The occupational health service system of Korea is customised based on the scale of enterprises. The large scale enterprises over 300 employees manage occupational health themselves, the medium scale ones between 50 and 299 employees manage themselves or entrust to the specialised institutions of health management. The public occupational health services, such as workers' health centres, provide technical support to the small scale enterprises under 50 employees. The occupational health activities in terms of policy, implementation, training and education etc. following the Seoul Statement are conducted progressively. Especially since the new government was launched, the occupational safety and health is emphasised to cover all workers.

\section{$1719 \mathrm{e}$ INTERCOUNTRY NETWORKING OCCUPATIONAL HEALTH SERVICES IN JAPAN AND ASIA}

${ }^{1}$ Toru Yoshikawa, ${ }^{2}$ Kazutaka Kogi. ${ }^{1}$ National Institute of Occupational Safety and Health, Kawasaki, Japan; ${ }^{2}$ Ohara Memorial Institute for Science of Labour, Tokyo, Japan

10.1136/oemed-2018-ICOHabstracts.641

The collaboration through inter-country networking in facilitating prevention-oriented programmes in occupational health services (OHS) is discussed. Experiences within our Asian regional networking of action-oriented approaches are examined to know types of support that can effectively facilitate the situation-based OHS including those for small-scale workplaces.

Commonly effective collaborative actions through the networking of action-oriented programme within OHS are reviewed. The networking has evolved since the 1990s to mutually support these activities for small enterprises, trade unions and health care workers and stress prevention. Attention is paid to support measures effective for facilitating practical workplace-level improvements and toolkits development.

The reviewed programmes have led to many work improvements in the different settings. The programmes apply locally tailored, action-oriented activities with the support of OHS teams. Prominent examples include Work Improvement in Small Enterprises (WISE) or similar methods for small workplaces, locally adjusted services and training in varied sectors and recent stress prevention interventions. The improvements achieved include many low-cost ones in work methods, physical environment and work organisation. Locally arranged networks of trainers are found instrumental in adjusting the methods to local situations. The collaborative actions of network partners are effective when they focus on the following aspects:

a. universally applicable simple procedures translated into locally practicable low-cost improvements addressing multiple factors;

b. participatory approaches building on local good practices; and

c. use of participatory action-oriented toolkits comprising checklists and training guides. 